\title{
A universal constant for semistable limit cycles
}

\author{
JOAN C. ARTÉS ${ }^{1}$, JAUME LLIBRE $^{1}$ and MARCO ANTONIO TEIXEIRA ${ }^{2}$ \\ ${ }^{1}$ Departament de Matemàtiques, Universitat Autònoma de Barcelona, \\ 08193 Bellaterra, Barcelona, Catalonia, Spain \\ ${ }^{2}$ Departamento de Matemática, Universidade Estadual de Campinas, \\ 13083-859, Campinas, SP, Brazil \\ E-mails: artes@mat.uab.cat / jllibre@mat.uab.cat / teixeira@ime.unicamp.br
}

\begin{abstract}
We consider one-parameter families of 2-dimensional vector fields $X_{\mu}$ having in a convenient region $R$ a semistable limit cycle of multiplicity $2 m$ when $\mu=0$, no limit cycles if $\mu \lesssim 0$, and two limit cycles one stable and the other unstable if $\mu \gtrsim 0$.

We show, analytically for some particular families and numerically for others, that associated to the semistable limit cycle and for positive integers $n$ sufficiently large there is a power law in the parameter $\mu$ of the form $\mu_{n} \approx C n^{\alpha}<0$ with $C, \alpha \in \mathbb{R}$, such that the orbit of $X_{\mu_{n}}$ through a point of $p \in R$ reaches the position of the semistable limit cycle of $X_{0}$ after given $n$ turns.

The exponent $\alpha$ of this power law depends only on the multiplicity of the semistable limit cycle, and is independent of the initial point $p \in R$ and of the family $X_{\mu}$. In fact $\alpha=-2 m /(2 m-1)$. Moreover the constant $C$ is independent of the initial point $p \in R$, but it depends on the family $X_{\mu}$ and on the multiplicity $2 m$ of the limit cycle $\Gamma$.
\end{abstract}

Mathematical subject classification: $58 \mathrm{~F} 14,58 \mathrm{~F} 21,58 \mathrm{~F} 30$.

Key words: semistable limit cycle, semistable fixed point, universal constant, power law.

\#CAM-233/10. Received: 06/VII/10. Accepted: 18/XI/10.

*The first two authors are partially supported by a MEC/FEDER grant BFM2008-03437, and a CIRIT grant number 2009SGR 410. The third author is partially supported by a grant FAPESP-2007/06896-5. All authors are also supported by the joint project CAPES-MECD grant HBP-2009-0025-PC. 


\section{Introduction and statement of the main results}

Let $X_{\mu}$ be an one-parameter family of 2-dimensional vector fields. An isolated periodic orbit of $X_{\mu}$ in the set of all periodic orbits of $X_{\mu}$ is called a limit cycle. For a definition of stable or unstable limit cycle, semistable limit cycle and multiplicity of a limit cycle, see for instance $[3,6]$.

Suppose that $X_{0}$ has a semistable limit cycle $\Gamma$, that $X_{\mu}$ for $\mu \lesssim 0$ has no limit cycles in a given annular neighborhood $A$ of $\Gamma$, and that $X_{\mu}$ for $\mu \gtrsim 0$ has two limit cycles near $\Gamma$ in $A$, one stable and the other unstable. So the family $X_{\mu}$ exhibits a bifurcation at $\mu=0$. We also assume that the flow of every $X_{\mu}$ with $\mu \lesssim 0$ enters into $A$ through one of its boundaries $B \approx \mathbb{S}^{1}=\mathbb{R} /(2 \pi \mathbb{R})$ and exits through the other. We denote by $R$ the annular subregion of $A$ limited by $B$ and $\Gamma$.

We take the annular region $R$ sufficiently narrow in such a way that for a point $p \in R$ we can choose as coordinates $(r, \theta)$, where $\theta$ is the angular variable along the periodic orbit $\gamma$ with $\theta \in \mathbb{S}^{1}$, and $r$ is the distance along the orthogonal segment to $\gamma$ through $p$.

We now fix a point $p=(r, \theta) \in R \backslash \Gamma$. For $\mu \lesssim 0$ the orbit $\gamma_{\mu}$ of $X_{\mu}$ through $p$ crosses $\gamma$ in positive time. We define $\mu_{n}$ as the value of $\mu \lesssim 0$ for which $\gamma_{\mu}$ reaches the point $q=(0, \theta+2 \pi n) \in \Gamma$, i.e. the orbit $\gamma_{\mu_{n}}$ starting at $p$ reaches $\Gamma$ at the point $q$ after doing exactly $n$ turns. In this way we have a sequence of increasing values of the parameter $\mu$ :

$$
\mu_{l}<\mu_{l+1}<\cdots<\mu_{n}<\cdots<0,
$$

tending to 0 . We have the following conjecture.

Conjecture 1. Assume that we have an one-parameter family of 2-dimensional vector fields $X_{\mu}$ satisfying all the previous assumptions stated in this section. Then for positive integers $n$ sufficiently large we have that

$$
\mu_{n} \approx C n^{\alpha} \quad \text { with } \quad \alpha=-\frac{2 m}{2 m-1},
$$

with $C \in \mathbb{R}$. Moreover the constant $C$ is independent of the initial point $p \in R$, but depends on the family $X_{\mu}$ and on the multiplicity $2 m$ of the limit cycle $\Gamma$. 
The expression $C n^{\alpha}$ for $\mu_{n}$ is called a power law with constant $C$ and exponent $\alpha$. Note that in particular the conjecture says that the exponent $\alpha$ depends only on the multiplicity of the semistable limit cycle $\Gamma$, and is independent of the initial point $p \in R$ and of the family $X_{\mu}$. In this sense the exponent $\alpha$ is a universal constant for the semistable limit cycles.

Conjecture 1 is supported by some analytical and numerical results. The analytical result is the following one.

Theorem 2. Let $(r, \theta)$ be the polar coordinates on the cylinder $\mathbb{R} \times \mathbb{S}^{1}$. Consider the one-parameter family of 2-dimensional vector fields

$$
X_{\mu}=(\dot{r}, \dot{\theta})=\left(k r^{2 m}-\mu, 1\right) \text { with } k>0 \text { and } \mu \in \mathbb{R} .
$$

Such a family for $\mu=0$ has the semistable limit cycle $r=0$ of multiplicity $2 m$. Then we have that $\mu_{n} \approx C n^{\alpha}$ where

$$
\alpha=-\frac{2 m}{2 m-1} \quad \text { and } \quad C=-k^{-\frac{1}{2 m-1}}\left(4 m \sin \left(\frac{\pi}{2 m}\right)\right)^{-\frac{2 m}{2 m-1}} .
$$

Theorem 2 is proved in Section 2.

Note that in Theorem 2 the constant $C$ depends on the vector fields $X_{\mu}$ through the constant $k$, and also depends on the multiplicity of the semistable limit cycle, but it is independent on the initial point $p \in R$ used for computing the values $\mu_{n}$. All this will be detailed in the proof of Theorem 2 .

The numerical results giving support to Conjecture 1 are described in Section 3 .

It easy to check that when $\mu_{n}$ follows a power law $C n^{\alpha}$ the value of the limit

$$
\lim _{n \rightarrow \infty} \frac{\mu_{n}-\mu_{n-1}}{\mu_{n+1}-\mu_{n}}
$$

is always equal to 1 . This quotient is the one studied by Feigembaum $[4,5]$ when he found the universal constant for a cascade of period doubling bifurcations. But for power laws this limit does not provide any information.

In this paper we have restricted our attention to one-parameter families of 2-dimensional vector fields $X_{\mu}$, but if we consider the return map $f_{0}: I \rightarrow I$ associated to the semistable limit cycle $\Gamma$ of $X_{0}$, and extend it to $X_{\mu}$ for $\mu$ in a 
neighborhood of $\mu=0$, we get an one-parameter family $f_{\mu}: I \rightarrow I$ of interval maps. Here $I$ is a small segment transversal to the flow of $X_{0}$ which intersects the semistable limit cycle $\Gamma$ at a point $q$ in the interior of $I$. We denote by $q_{i}$ with $i=1,2$ the endpoints of the segment $I$.

By construction the family of interval maps $f_{\mu}$ has a semistable fixed point of multiplicity $2 m$ at $\mu=0$, no fixed points if $\mu \lesssim 0$, and two fixed points one stable and the other unstable if $\mu \gtrsim 0$. We can translate the results obtained for the studied families of 2-dimensional vector fields $X_{\mu}$, to their corresponding families of interval maps $f_{\mu}$.

We denote by $R$ the subsegment of $I$ with endpoints either $q_{1}$ and $q$, or $q$ and $q_{2}$, such that the $\omega$-limit of all the points of $R$ under $f_{0}$ are equal to $q$. As for $X_{\mu}$ we get for the semistable fixed point $q$ and for positive integers $n$ sufficiently large a power law in the parameter $\mu$ of the form $\mu_{n} \approx C n^{\alpha}<0$ with $C, \alpha \in \mathbb{R}$, such that the orbit of $f_{\mu_{n}}$ through a point of $p \in R$ reaches the position $q$ of the semistable fixed point of $f_{0}$ after $n$ iterates. Translating Conjecture 1 from the family of vector fields $X_{\mu}$ to the family of interval maps $f_{\mu}$ we have the conjecture.

Conjecture 3. Assume that we have an one-parameter family of interval maps $f_{\mu}$ satisfying the previous assumptions. Then for positive integers $n$ sufficiently large we have that

$$
\mu_{n} \approx C n^{\alpha} \quad \text { with } \quad \alpha=-\frac{2 m}{2 m-1},
$$

with $C \in \mathbb{R}$. Moreover $C$ is independent of the initial point $p \in R$, but depends on the family $f_{\mu}$ and on the multiplicity $2 m$ of the semistable fixed point $q$.

Some open questions are:

(1) How to prove Conjectures 1 or 3 ?

(2) Does there exist a similar result or conjecture on the stable manifold of semistable limit cycles for one-parameter families of vector fields in dimension larger than 2 ?

(3) Does there exist a similar result or conjecture on the stable manifold of semistable fixed points for one-parameter families of functions in dimension larger than 1 ? 


\section{Proof of Theorem 2}

The solutions of the vector field $X_{\mu}=(\dot{r}, \dot{\theta})=\left(k r^{2 m}-\mu, 1\right)$ with $k>0$ on the cylinder $(r, \theta) \in \mathbb{R} \times \mathbb{S}^{1}$ can be studied solving the differential equation

$$
\frac{d r}{d \theta}=k r^{2 m}-\mu \text {. }
$$

Clearly for $\mu<0$ we have that $d r / d \theta>0$, therefore the differential equation (1) has no periodic orbits. For $\mu=0$ the unique periodic orbit is $r(\theta)=0$, that is a semistable limit cycle of multiplicity $2 \mathrm{~m}$. It attracts the orbits of the half-cylinder $r<0$, and repels the orbits of the other half-cylinder $r>0$. Finally when $\mu>0$ equation (1) has exactly two limit cycles, namely $r_{ \pm}(\theta)=$ $\pm(\mu / k)^{1 /(2 m)}$. The limit cycle $r_{-}(\theta)$ is stable, and the limit cycle $r_{+}(\theta)$ is unstable.

The differential equation (1) is of separable variables, so we can solve it and get that the solution $r(\theta)$ such that $r(0)=r_{0}$ is

$$
\theta=\frac{r_{0}}{\mu} F\left(\frac{1}{2 m}, 1 ; 1+\frac{1}{2 m} ; \frac{k r_{0}^{2 m}}{\mu}\right)-\frac{r(\theta)}{\mu} F\left(\frac{1}{2 m}, 1 ; 1+\frac{1}{2 m} ; \frac{k r(\theta)^{2 m}}{\mu}\right),
$$

where $F(a, b ; c ; x)$ is the hypergeometric function

$$
F(a, b ; c ; x)=\sum_{k=0}^{\infty} \frac{(a)_{k}(b)_{k}}{(c)_{k}} \frac{x^{k}}{k !} .
$$

Here we have used the notation

$$
(a)_{k}= \begin{cases}1 & \text { if } k=0, \\ a(a+1)(a+2) \cdots(a+k-1) & \text { if } k>0 .\end{cases}
$$

For more details on this hypergeometric function see [1].

In order to compute $\mu_{n}$ we must find the value of $\mu$ satisfying equation (2) when $\theta=2 \pi n$ and $r(2 \pi n)=0$ for a given value of $r_{0}<0$, i.e. we must solve

$$
\theta=\frac{r_{0}}{\mu_{n}} F\left(\frac{1}{2 m}, 1 ; 1+\frac{1}{2 m} ; \frac{k r_{0}^{2 m}}{\mu_{n}}\right),
$$

with respect to $\mu_{n}$. Developing equation (3) in Laurent series with respect to the small parameter $\mu_{n}$ we obtain

$$
2 \pi n=\frac{\pi}{2 m} \csc \left(\frac{\pi}{2 m}\right) k^{-\frac{1}{2 m}}\left(-\mu_{n}\right)^{\frac{1-2 m}{2 m}}-\frac{r_{0}^{1-2 m}}{k(1-2 m)}+O\left(\mu_{n}\right) .
$$


Since $\mu_{n} \rightarrow 0$ when $n \rightarrow \infty$, for $n$ sufficiently large we have that

$$
2 \pi n \approx \frac{\pi}{2 m} \csc \left(\frac{\pi}{2 m}\right) k^{-\frac{1}{2 m}}\left(-\mu_{n}\right)^{\frac{1-2 m}{2 m}} .
$$

From this expression the power law stated in Theorem 2 follows.

\section{Numerical results for vector fields}

In this section we shall compute the sequence $\left(\mu_{n}\right)$ numerically for several different kind of planar polynomial vector fields, and we shall see that all the sequences obtained provide support to Conjecture 1.

Once we have the numerical values of the sequence $\left(\mu_{n}\right)$ if for $n$ sufficiently large this sequence follows a power law $C n^{\alpha}$, we can compute $\alpha$ and $C$ as follows

$$
\begin{aligned}
& \alpha=\lim _{n \rightarrow \infty} \alpha_{n}=\lim _{n \rightarrow \infty} \frac{\log \left|\mu_{n+1}\right|-\log \left|\mu_{n}\right|}{\log (n+1)-\log n}, \\
& C=-\exp \left(\lim _{n \rightarrow \infty} C_{n}\right)=-\exp \left(\lim _{n \rightarrow \infty}\left(\log \left|\mu_{n}\right|-\alpha \log n\right)\right) .
\end{aligned}
$$

We divide the rest of this section in five subsections. In every one we study Conjecture 1 for a different family of polynomial vector fields $X_{\mu}$.

\subsection{A polynomial vector field of degree 5}

In all this subsection $X=(P, Q)$ will denote the following polynomial vector field of degree 5

$$
\begin{aligned}
X(x, y) & =(P(x, y), Q(x, y)) \\
& =\left(-y+x\left(x^{2}+y^{2}-1\right)^{2}, x+y\left(x^{2}+y^{2}-1\right)^{2}\right) .
\end{aligned}
$$

Since in polar coordinates $(r, \theta)$, defined by $(x, y)=(r \cos \theta, r \sin \theta)$, the vector field becomes

$$
X(r, \theta)=\left(r\left(r^{2}-1\right)^{2}, 1\right),
$$

it follows that the phase portrait of $X$ is formed by an unstable focus at the origin $O$ and a semistable limit cycle $\Gamma$ at $r=1$ of multiplicity 2, see Figure 1. If an orbit is contained in the annulus $0<r<1$, then its $\alpha$-limit is $O$ and 


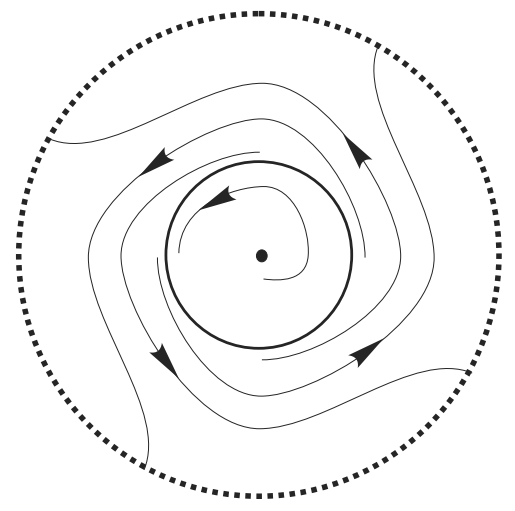

Figure 1 - Phase portrait in the Poincaré disc of the vector field $X$.

its $\omega$-limit is $\Gamma$. If an orbit is contained in the annulus $r>1$, then its $\alpha$-limit is $\Gamma$ and its $\omega$-limit is at infinity. Moreover, in the Poincaré compactification the circle of infinity is formed by a continuum of singular points. For more details on the Poincaré compactification see Chapter 5 of [3]. When we work with the Poincaré compactification of a polynomial vector field we shall use the notation introduced in that chapter. Thus the continuum of singular points at infinity follows from the fact that the polynomials $F\left(z_{1}\right)$ and $G\left(z_{1}\right)$ (defined in the mentioned Chapter 5) and whose zeros provide the singular points at infinity are identically zero.

See Chapter 7 of [3] for a summary on the dependence of the limit cycles with respect to the parameter of a rotated family of vector fields. The family of vector fields

$$
X_{\mu}(x, y)=(P(x, y), Q(x, y, \mu))=(P(x, y), Q(x, y)+\mu P(x, y)),
$$

forms a rotated parameter family with respect to the parameter $\mu \in \mathbb{R}$, because the singular points of $X_{\mu}$ remain the same for all $\mu$, the determinant

$$
\left|\begin{array}{ll}
P(x, y) & Q\left(x, y, \mu_{1}\right) \\
P(x, y) & Q\left(x, y, \mu_{2}\right)
\end{array}\right|=\left(\mu_{2}-\mu_{1}\right) P(x, y)^{2} \geq 0
$$

if $\mu_{1}<\mu_{2}$; and the equality cannot hold on an entire periodic orbit of $X_{\mu}$ with $\mu=\mu_{i}$, for $i=1,2$. To verify this, it is sufficient to see that the curve $P(x, y)=0$ cannot contain an oval. Since in polar coordinates this curve becomes the origin union the curve $\tan \theta=\left(r^{2}-1\right)^{2}$, it follows that it cannot 
contain an oval surrounding the origin, the unique singular point of the system, because $\tan \theta$ cannot take negative values. We note that in the definition of our rotated family of vector fields the interval coincides with the real line.

Of course $X_{0}=X$. So, by the semistable property (see Chapter 7 of [3]), $\mu=0$ is a bifurcation value of the parameter $\mu$ because for $\mu \gtrsim 0$ there are two limit cycles (one stable and the other unstable) since the multiplicity of the semistable limit cycle $x^{2}+y^{2}=1$ of the vector field $X$ is two, and for $\mu \lesssim 0$ there are no limit cycles.

Using the program $\mathrm{P} 4$ see the last chapter of [3] (which allows to draw the compactified phase portrait of a polynomial vector field in the Poincaré disc), we know that for $\mu<0$ small (from now on small means sufficiently small) the vector field $X_{\mu}$ has no limit cycles in the whole plane $\mathbb{R}^{2}$.

We are only interested in studying the phase portrait of $X_{\mu}$ for $\mu<0$ and small. For $\mu<0$ and small every orbit starting at a point $(x, y)$ with $x^{2}+$ $y^{2}>0$ goes to infinity in forward time giving finitely many turns around the origin, and goes to the origin in backwards time giving infinitely many turns around it. Now we will prove this claim.

First we study the infinity of $X_{\mu}$ with $\mu<0$ and small. Using the notation of Chapter 5 of [3] we have that

$$
F\left(z_{1}\right)=b\left(1+z_{1}^{2}\right)^{2}, \quad G\left(z_{1}\right)=-b z_{1}^{2}\left(1+z_{1}^{2}\right)^{2} .
$$

Therefore the unique infinite singular points of $X_{\mu}$ are the origins of the local charts $U_{2}$ and $V_{2}$; i.e. the endpoints of the $y$-axis. The eigenvalues at these singular points are -1 and 0 . Therefore, applying Theorem 2.19 of [3], we know that these singular points only can be a node, a saddle or a saddle-node with topological indices $1,-1$ and 0 , respectively. Of course, since the local phase portrait at these two diametrally opposite singular points at infinity are symmetric with respect the origin of the Poincaré sphere $\mathbb{S}^{2}$ (due to the construction of the Poincaré compactification), they have the same topological index.

By the Poincaré-Hopf Theorem (see, for instance, Chapter 6 of [3]), if $p\left(X_{\mu}\right)$ on $\mathbb{S}^{2}$ has finitely many singular points (as it is the case for $\mu<0$ ), then the sum of all their topological indices is 2 . Now, the sum of the topological indices of the finite singular point of $X_{\mu}$ is 1 , because it is a focus for 
$\mu<0$ and small. Therefore the sum of the indices of the Poincare compactification $p\left(X_{\mu}\right)$ in $\mathbb{S}^{2} \backslash \mathbb{S}^{1}$ is 2 . Consequently the sum of the indices of the two infinite singular points must be zero. Hence, the indices of those points are 0 , and consequently they are saddle-nodes. Again, using Theorem 2.19 of [3], we get that their local phase portraits are those of Figure 2.

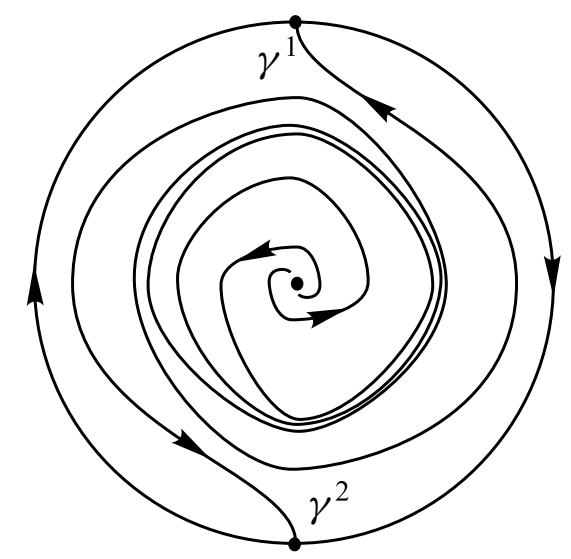

Figure 2 - Phase portrait in the Poincaré disc of the vector field $X_{\mu}$ with $\mu<0$ small.

In short we know for all the singular points of $X_{\mu}$, located at infinity or not, their local phase portraits, we also know that the vector fields $X_{\mu}$ form a rotated family with respect to $\mu$, consequently the phase portrait of $X_{\mu}$ for $\mu<0$ and small has no limit cycles near $x^{2}+y^{2}=1$ (the unique possible place for the limit cycles). Hence the phase portrait of $X_{\mu}$ for $\mu<0$ and small is the one given in Figure 2. Therefore the claim is proved.

From Figure 2 it is clear that $X_{\mu}$ for $\mu<0$ and small has only two separatrices $\gamma_{\mu}^{1}$ and $\gamma_{\mu}^{2}$ contained in $\mathbb{R}^{2}$, the stable ones of the saddle-nodes located at the origins of the local charts $U_{2}$ and $V_{2}$; i.e. at the endpoints of the $y$-axis. Here a separatrix is an orbit in the boundary of a hyperbolic sector, see for more details [3]. Of course, the $\omega$-limit of $\gamma_{b}^{1}$ is the origin of $U_{2}$, while the $\omega$-limit of $\gamma_{b}^{2}$ is the origin of $V_{2}$, and their $\alpha$-limit is the origin $O$ of $\mathbb{R}^{2}$.

Since $X_{\mu}$ for $\mu=0$ has a semistable limit cycle $\Gamma$ at $x^{2}+y^{2}=1$, for $\mu<0$ and $\mu$ small it follows that the number of turns of $\gamma_{\mu}^{1}$ or $\gamma_{\mu}^{2}$ around $O$ from the infinity to a point of the annulus $R=\left\{(x, y): 1 / 2 \leq x^{2}+y^{2}<1\right\}$ increases when $\mu$ increases, and tends to $\infty$ when $\mu \nearrow 0$. 
Now we fix a point $p_{0}=\left(x_{0}, y_{0}\right) \in R$ and we shall use the separatrix $\gamma_{\mu}=\gamma_{\mu}^{1}$ run in backward time for computing the sequences $\left(\mu_{n}\right)$. More precisely, we compute the intersection point $r_{\mu}$ of separatrix $\gamma_{\mu}$ with the circle $x^{2}+y^{2}=1$, i.e. with $\Gamma$. After we look for the value $\mu_{n}$ such $\gamma_{\mu}$ runs in backward time from $r_{\mu}$ to the point $p_{0}$ given $n$ turns and less than $n+1$. Due to the existence of the semistable limit cycle for $\mu=0$, there is a sequence of increasing values

$$
\mu_{1}<\mu_{2}<\cdots<\mu_{n}<\cdots<0,
$$

tending to 0 for which $\gamma_{\mu_{n}}$ passes through $p_{0}$ exactly after doing $n$ turns around $O$ and less than $n+1$. Of course, these $\mu_{n}$ does not coincide exactly with the ones defined in Section 1, but their differences tend to zero when $n$ tends to infinity.

\begin{tabular}{|c|c|c|c|}
\hline$n$ & $\mu_{n}$ & $\alpha_{n}$ & $C_{n}$ \\
\hline 1 & $-2,881632841 \cdot 10^{-2}$ & & \\
2 & $-7,901508033 \cdot 10^{-3}$ & $-1,866687$ & $-0,028816$ \\
3 & $-3,577475828 \cdot 10^{-3}$ & $-1,954289$ & $-0,030620$ \\
4 & $-2,021534899 \cdot 10^{-3}$ & $-1,984136$ & $-0,031641$ \\
5 & $-1,294524787 \cdot 10^{-3}$ & $-1,997429$ & $-0,032229$ \\
& $\ldots \ldots \ldots \ldots$ & $\ldots \ldots$ & $\ldots \ldots$ \\
401 & $-1,957431584 \cdot 10^{-7}$ & $-2,001752$ & $-0,031649$ \\
402 & $-1,947672191 \cdot 10^{-7}$ & $-2,001812$ & $-0,031661$ \\
403 & $-1,937985601 \cdot 10^{-7}$ & $-2,001809$ & $-0,031660$ \\
404 & $-1,928371089 \cdot 10^{-7}$ & $-2,001805$ & $-0,031660$ \\
405 & $-1,918827943 \cdot 10^{-7}$ & $-2,001802$ & $-0,031659$ \\
& $\ldots \ldots \ldots \ldots$. & $\ldots \ldots$ & $\ldots \ldots$ \\
\hline
\end{tabular}

Table $1-$ Values of $\mu_{n}$ for the point $p_{0}=(0,3 / 4)$.

We estimate the values $\mu_{n}$ and (4) for different points $p_{0}$, and there is numerical evidence that the limit $\alpha$ of the sequence $\alpha_{n}$ is $-2=-2 / 1$, independently of the point $p_{0}$. More precisely, for the point $p_{0}=(0,3 / 4)$ the values of $\mu_{n}, \alpha_{n}$ and $C_{n}$ appear in Table 1 , and for $p_{0}=(0,1 / 2)$ in Table 2 . We have also done the study starting from the point $(0,7 / 8)$ but we do not add the table here. We will just summarize the results of all them in Table 5 at the 
end of the paper. Just to note that for this last case $\alpha_{404}=-2,000650974$ and $C_{404}=-0,031405$.

Similar results to those of Tables 1 and 2 are obtained if we do the computations with the other separatrix or with any other initial point $p_{0}$.

\begin{tabular}{|c|c|c|c|}
\hline$n$ & $\mu_{n}$ & $\alpha_{n}$ & $C_{n}$ \\
\hline 1 & $-3,988226061 \cdot 10^{-2}$ & & \\
2 & $-9,468261118 \cdot 10^{-3}$ & $-2,074576$ & $-0,039882$ \\
3 & $-4,046251050 \cdot 10^{-3}$ & $-2,096739$ & $-0,040500$ \\
4 & $-2,218353717 \cdot 10^{-3}$ & $-2,089200$ & $-0,040166$ \\
5 & $-1,394597364 \cdot 10^{-3}$ & $-2,080094$ & $-0,039662$ \\
& $\ldots \ldots \ldots \ldots$ & $\ldots \ldots$ & $\ldots \ldots$ \\
401 & $-1,959227559 \cdot 10^{-7}$ & $-2,002671$ & $-0,031853$ \\
402 & $-1,949454750 \cdot 10^{-7}$ & $-2,002729$ & $-0,031864$ \\
403 & $-1,939754875 \cdot 10^{-7}$ & $-2,002723$ & $-0,031863$ \\
404 & $-1,930127212 \cdot 10^{-7}$ & $-2,002718$ & $-0,031862$ \\
405 & $-1,920571044 \cdot 10^{-7}$ & $-2,002712$ & $-0,031861$ \\
& $\ldots \ldots \ldots \ldots .$. & $\ldots \ldots$ & $\ldots \ldots$ \\
\hline
\end{tabular}

Table 2 - Values of $\mu_{n}$ for the point $p_{0}=(0,1 / 2)$.

Looking to these results one could think that $\alpha$ may be -2 for all the considered cases independently of the initial point, and also we can think that the constant $C$ is independent of the initial point $p_{0}$. When we compare this constant $C$ with the ones obtained for different families of vector fields, we will see that it must depend on the family and/or the multiplicity. This dependence becomes clear in the analytic example of Section 2. But again the constant $C$ looks independent of the initial point $p_{0}$, this can be see clearly in Table 5 where we summarize the results of the first three subsections.

\subsection{A polynomial vector field of degree 9}

In this subsection $X=(P, Q)$ will denote the polynomial vector field of degree 9 given by

$$
\begin{aligned}
X(x, y) & =(P(x, y), Q(x, y)) \\
& =\left(-y+x\left(x^{2}+y^{2}-1\right)^{4}, x+y\left(x^{2}+y^{2}-1\right)^{4}\right) .
\end{aligned}
$$




\begin{tabular}{|c|c|c|c|}
\hline$n$ & $\mu_{n}$ & $\alpha_{n}$ & $C_{n}$ \\
\hline 1 & $-1,233572 \cdot 10^{-1}$ & & \\
2 & $-4,656607 \cdot 10^{-2}$ & $-1,405490$ & $-0,123357$ \\
3 & $-2,604312 \cdot 10^{-2}$ & $-1,433214$ & $-0,125751$ \\
4 & $-1,722170 \cdot 10^{-2}$ & $-1,437642$ & $-0,126364$ \\
5 & $-1,249909 \cdot 10^{-2}$ & $-1,436358$ & $-0,126139$ \\
& $\ldots \ldots \ldots \ldots$ & $\ldots \ldots$ & $\ldots \ldots$ \\
401 & $-2,841841 \cdot 10^{-5}$ & $-1,355451$ & $-0,095623$ \\
402 & $-2,832239 \cdot 10^{-5}$ & $-1,355387$ & $-0,095587$ \\
403 & $-2,822694 \cdot 10^{-5}$ & $-1,355409$ & $-0,095600$ \\
404 & $-2,813205 \cdot 10^{-5}$ & $-1,355389$ & $-0,095588$ \\
405 & $-2,803771 \cdot 10^{-5}$ & $-1,355368$ & $-0,095576$ \\
& $\ldots \ldots \ldots \ldots .$. & $\ldots \ldots$ & $\ldots \ldots$ \\
\hline
\end{tabular}

Table 3 - Values of $\mu_{n}$ for the point $p_{0}=(0,1 / 2)$.

In polar coordinates $(r, \theta)$ the vector field becomes

$$
X(r, \theta)=\left(r\left(r^{2}-1\right)^{4}, 1\right) .
$$

Therefore the phase portrait of $X$ is formed by an unstable focus $O$ at the origin and a semistable limit cycle $\Gamma$ at $r=1$ of multiplicity 4 . This phase portrait is topologically equivalent to the one of Figure 1.

Repeating the arguments of Subsection 3.1, we obtain that the vector fields $X_{\mu}(x, y)=(P(x, y), Q(x, y)+\mu P(x, y))$ form a rotated family with respect to the parameter $\mu \in \mathbb{R}$. Again, $X_{0}=X$, and $\mu=0$ is a bifurcation value for the parameter $\mu$ in such a way that for $\mu<0$ and small the vector field $X_{\mu}$ has no limit cycles. For $\mu<0$ and small every orbit starting at a point $(x, y)$ with $x^{2}+y^{2}>0$ goes to infinity in forward time giving finitely many turns around the origin and goes to the origin in backward time giving infinitely many turns around it, see Figure 2. From this figure, it is clear that $X_{\mu}$ for $\mu<0$ and small has only two separatrices $\gamma_{\mu}^{1}$ and $\gamma_{\mu}^{2}$ contained in $\mathbb{R}^{2}$.

We fix a point $p_{0}=\left(x_{0}, y_{0}\right)$ in $R=\left\{(x, y): 1 / 2 \leq x^{2}+y^{2}<1\right\}$ and we compute the sequence $\left(\mu_{n}\right)$ as in the previous subsection. 
Again for different points $p_{0}$, there is numerical evidence that the limit of $\alpha$ as $n \rightarrow \infty$ can be $-4 / 3=-1.33333 \ldots$ depending only on the multiplicity 4 of $\Gamma$. If we start from $p=(0,3 / 4)$ (respectively $p=(0,7 / 8))$ we get that $\alpha_{404}=-1,353415 \ldots$ (respectively $\alpha_{404}=-1,345133 \ldots$ ). Increasing the multiplicity of the semistable limit cycle increases the time of the computations for obtaining the same precision in the values of $\mu_{n}$.

\subsection{A polynomial vector field of degree 13}

In this subsection $X=(P, Q)$ will denote the polynomial vector field of degree 13 given by

$$
\begin{aligned}
X(x, y) & =(P(x, y), Q(x, y)) \\
& =\left(-y+x\left(x^{2}+y^{2}-1\right)^{6}, x+y\left(x^{2}+y^{2}-1\right)^{6}\right) .
\end{aligned}
$$

In polar coordinates $(r, \theta)$ the vector field becomes

$$
X(r, \theta)=\left(r\left(r^{2}-1\right)^{6}, 1\right)
$$

Therefore the phase portrait of $X$ is formed by an unstable focus $O$ at the origin and a semistable limit cycle $\Gamma$ at $r=1$ of multiplicity 6 . This phase portrait is topologically equivalent to the one of Figure 1.

We construct the family of vector fields $X_{\mu}$ with $X_{0}=X$ as in Subsection 3.2, and compute the sequence $\left(\mu_{n}\right)$ as in that subsection.

Again for different points $p_{0}$, there is numerical evidence that the limit of $\mu_{n}$ is $-6 / 5=-1.2$ as $n \rightarrow \infty$ depending only on the multiplicity 6 of the semistable limit cycle, because $\alpha_{404}=-1,234375 \ldots$ If we start from $p=(0,3 / 4)$ (respectively $p=(0,7 / 8))$ we get that $\alpha_{404}=-1,228354 \ldots$ (respectively $\alpha_{404}=-1,159198 \ldots$.. . Now the differences between them become more evident, but we think that if we go to bigger values in $n$ for $\alpha_{n}$, when the multiplicity increases these differences in $\alpha_{n}$ changing the initial point $p_{0}$ will disappear. In any case increasing $n$ and the multiplicity the time for the computations increases strongly.

Gathering all the results from Subsections 3.1 to 3.3 we get Table 5 . 


\begin{tabular}{|c|c|c|c|}
\hline$n$ & $\mu_{n}$ & $\alpha_{n}$ & $C_{n}$ \\
\hline 1 & $-1,684320 \cdot 10^{-1}$ & & \\
2 & $-7,177137 \cdot 10^{-2}$ & $-1,230686$ & $-0,168432$ \\
3 & $-4,299420 \cdot 10^{-2}$ & $-1,263784$ & $-0,172341$ \\
4 & $-2,977758 \cdot 10^{-2}$ & $-1,276789$ & $-0,174821$ \\
5 & $-2,236696 \cdot 10^{-3}$ & $-1,282453$ & $-0,176199$ \\
& $\ldots \ldots \ldots \ldots$ & $\ldots \ldots$ & $\ldots \ldots$ \\
401 & $-8,837415 \cdot 10^{-5}$ & $-1,234466$ & $-0,144483$ \\
402 & $-8,810286 \cdot 10^{-5}$ & $-1,234404$ & $-0,144430$ \\
403 & $-8,783307 \cdot 10^{-5}$ & $-1,234421$ & $-0,144444$ \\
404 & $-8,756478 \cdot 10^{-5}$ & $-1,234398$ & $-0,144425$ \\
405 & $-8,729798 \cdot 10^{-5}$ & $-1,234375$ & $-0,144405$ \\
& $\ldots \ldots \ldots \ldots$ & $\ldots \ldots$ & $\ldots \ldots$ \\
\hline
\end{tabular}

Table 4 - Values of $\mu_{n}$ for the point $p_{0}=(0,1 / 2)$.

\begin{tabular}{|c|c|c|c|c|}
\hline & \multicolumn{2}{|c|}{$\alpha$} & \multicolumn{2}{c|}{$\mathrm{C}$} \\
\hline Mult. 2 & $\gamma_{\mu}^{1}$ & $\gamma_{\mu}^{2}$ & $\gamma_{\mu}^{1}$ & $\gamma_{\mu}^{2}$ \\
\hline$p=(0,1 / 2)$ & $-2,002706$ & $-2,000427$ & $-0,031860$ & $-0,031343$ \\
$p=(0,3 / 4)$ & $-2,001798$ & $-1,999850$ & $-0,031658$ & $-0,031217$ \\
$p=(0,7 / 8)$ & $-2,000651$ & $-1,999054$ & $-0,031405$ & $-0,031043$ \\
\hline Mult. 4 & & & & \\
\hline$p=(0,1 / 2)$ & $-1,355347$ & $-1,347251$ & $-0,095564$ & $-0,128737$ \\
$p=(0,3 / 4)$ & $-1,353415$ & $-1,344790$ & $-0,094281$ & $-0,126525$ \\
$p=(0,7 / 8)$ & $-1,345133$ & $-1,327778$ & $-0,088967$ & $-0,112284$ \\
\hline Mult. 6 & & & & \\
\hline$p=(0,1 / 2)$ & $-1,234375$ & & $-0,144405$ & \\
$p=(0,3 / 4)$ & $-1,228354$ & & $-0,138444$ & \\
$p=(0,7 / 8)$ & $-1,159198$ & & $-0,084398$ & \\
\hline
\end{tabular}

Table 5 - Values of $\alpha$ and $C$ for the cases studied in Subsections 3.1-3.3. 


\subsection{A polynomial vector field of degree 7}

In order to not limit our study to a single family of phase portraits with a multiple semistable limit cycles, we will study now a completely different system which has more singular points than the origin.

In this subsection $X=(P, Q)$ will denote the polynomial vector field of degree 7 given by

$$
\begin{aligned}
P= & 4 x+2 x^{2}-8 x^{3}-4 x^{4}+4 x^{5}+2 x^{6}-4 y-4 x y+2 x^{2} y \\
& +8 x^{3} y-x^{4} y-4 x^{5} y-2 x^{6} y+6 y^{2}-8 x y^{2}-12 x^{2} y^{2}+8 x^{3} y^{2} \\
& +10 x^{4} y^{2}+2 y^{3}+8 x y^{3}-2 x^{2} y^{3}-8 x^{3} y^{3}-6 x^{4} y^{3}-8 y^{4}+4 x y^{4} \\
& +14 x^{2} y^{4}-y^{5}-4 x y^{5}-6 x^{2} y^{5}+6 y^{6}-2 y^{7}, \\
Q= & 4 x+2 x^{2}-2 x^{3}-4 x^{4}+x^{5}+2 x^{6}+2 x^{7}+4 y-4 x y-8 x^{2} y \\
& +4 x^{3} y+4 x^{4} y-4 x^{5} y-2 y^{2}-2 x y^{2}+2 x^{3} y^{2}+2 x^{4} y^{2}+6 x^{5} y^{2} \\
& -8 y^{3}+4 x y^{3}+8 x^{2} y^{3}-8 x^{3} y^{3}+4 y^{4}+x y^{4}-2 x^{2} y^{4}+6 x^{3} y^{4} \\
& +4 y^{5}-4 x y^{5}-2 y^{6}+2 x y^{6} .
\end{aligned}
$$

It is easy to check that

$$
\begin{aligned}
H= & \frac{1}{x^{2}+y^{2}-1}+2 \arctan \left(\frac{2-y}{x}\right)+2 \arctan \left(\frac{y}{x}\right) \\
& -\log \left(\left(x^{2}+(y-2)^{2}\right)\left(x^{2}+y^{2}\right)\right)
\end{aligned}
$$

is a first integral of $X$; i.e.

$$
\frac{\partial H}{\partial x} P+\frac{\partial H}{\partial y} Q=0
$$

and consequently $H$ is constant on the orbits of the vector field $X$. This first integral or the program $\mathrm{P} 4$ allows to show that the phase portrait of $X$ in the Poincare disc is the one of Figure 3. We note that $X$ has exactly three singular points, an unstable focus at the origin $(0,0)$, a stable focus at $(0,2)$, and a saddle at

$$
S=(-0.600233563294595 \ldots, 1.399766436705378 \ldots) .
$$




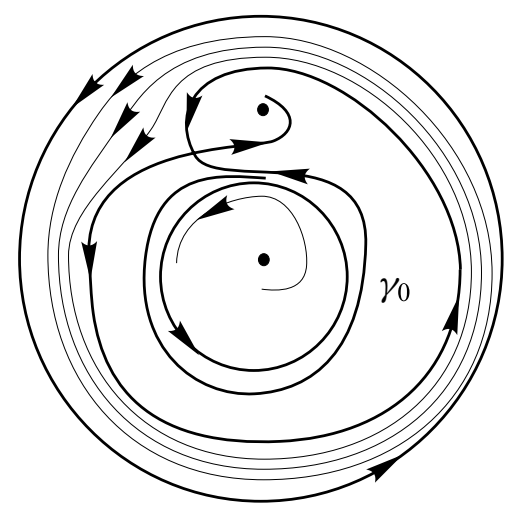

Figure 3 - Phase portrait in the Poincaré disc of the vector field $X$.

Since $f=x^{2}+y^{2}-1$ satisfies the equality

$$
\frac{\partial f}{\partial x} P+\frac{\partial f}{\partial y} Q=4(x-y+2)\left(x^{2}+y^{2}\right) f^{2},
$$

it follows that $f=0$ is an algebraic solution of $X$. Due to the fact that on $f=0$ there are no singular points, $f=0$ is a periodic orbit. Using the first integral we can see that it is isolated in the set of all periodic orbits, so it is a limit cycle, that we denote by $\Gamma$. Again, using the first integral, we can check that $\Gamma$ is a semistable limit cycle. Using results of [2] we can show that $\Gamma$ has multiplicity 2 .

Repeating the arguments of Subsection 3.1, we obtain that the vector fields $X_{\mu}(x, y)=(P(x, y), Q(x, y)+\mu P(x, y))$ form a rotated family with respect to the parameter $\mu \in \mathbb{R}$. Again, $X_{0}=X$, and $\mu=0$ is a bifurcation value for the parameter $\mu$ in such a way that for $b \mu<0$ and small the vector field $X_{\mu}$ has no limit cycles. By the non-intersection property (see Chapter 7 of [3]), there are no periodic orbits of $X_{\mu}$ in the region occupied by the period annulus around the infinity of system $X_{0}$.

In fact using the program P4 we see that the phase portrait of $X_{\mu}$ for $\mu<0$ and small is the one given in Figure 4. For these vector fields the infinity is a periodic orbit, this follows using Chapter 5 of [3] and checking that there are no infinite singular points.

Looking at Figure 4, we denote by $\gamma_{0}$ the stable separatrix of the saddle $S$ whose $\alpha$-limit for $X_{0}$ is the semistable limit cycle $f=0$. Then, for $\mu<0$ and 
small, we denote by $\gamma_{\mu}$ the stable separatrix of the saddle $S$ whose $\alpha$-limit for $X_{\mu}$ is the origin and such that $\gamma_{\mu}$ tends to $\gamma_{0}$ when $\mu \nearrow 0$, see Figure 4.

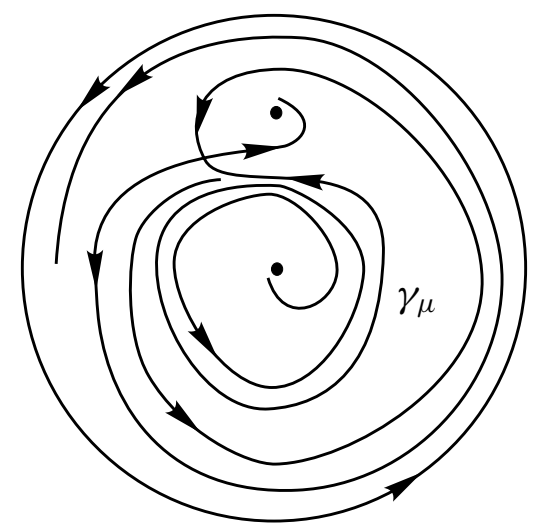

Figure 4 - Phase portrait in the Poincaré disc of the vector field $X_{\mu}$ with $\mu<0$ and small.

\begin{tabular}{|c|c|c|c|}
\hline$n$ & $\mu_{n}$ & $\alpha_{n}$ & $C_{n}$ \\
\hline 1 & $-3,794376 \cdot 10^{-2}$ & & \\
2 & $-8,410108 \cdot 10^{-3}$ & $-2,173666$ & $-0,037944$ \\
3 & $-3,648280 \cdot 10^{-3}$ & $-2,059804$ & $-0,035064$ \\
4 & $-2,027845 \cdot 10^{-3}$ & $-2,041427$ & $-0,034363$ \\
5 & $-1,288488 \cdot 10^{-3}$ & $-2,032344$ & $-0,033933$ \\
& $\ldots \ldots \ldots \ldots$ & $\ldots \ldots$ & $\ldots \ldots$ \\
401 & $-1,944170 \cdot 10^{-7}$ & $-2,000366$ & $-0,031331$ \\
402 & $-1,934507 \cdot 10^{-7}$ & $-2,000436$ & $-0,031344$ \\
403 & $-1,924917 \cdot 10^{-7}$ & $-2,000362$ & $-0,031330$ \\
404 & $-1,915398 \cdot 10^{-7}$ & $-2,000384$ & $-0,031335$ \\
405 & $-1,905949 \cdot 10^{-7}$ & $-2,000427$ & $-0,031343$ \\
& $\ldots \ldots \ldots \ldots$. & $\ldots \ldots$ & $\ldots \ldots$ \\
\hline
\end{tabular}

Table 6 - Values of $B_{n}$ for the point $p_{0}=(0,1 / 2)$.

We fix a point $p_{0}=\left(x_{0}, y_{0}\right)$ in $R=\left\{(x, y): 1 / 2 \leq x^{2}+y^{2}<1\right\}$, and use the separatrix $\gamma_{\mu}$ as we have used the separatrix $\gamma_{\mu}^{1}$ in Subsection 3.1 for computing the sequence $\left(\mu_{n}\right)$. 
For different points $p_{0}$ there is numerical evidence that the limit of $\mu_{n}$ is -2 as $n \rightarrow \infty$ independently of the point $p_{0}$, because $\alpha_{404}=-2,000427 \ldots$. If we start from $p=(0,3 / 4)$ (respectively $p=(0,7 / 8))$ we get that $\alpha_{404}=$ $-1,999850 \ldots$ (respectively $\alpha_{404}=-1,999054 \ldots$ ). Again these numbers indicate that $\alpha$ seems to be independent of the differential system we take, and only depends on the multiplicity of the semistable limit cycle.

\subsection{A polynomial vector field of degree 11}

Finally we take a new example similar to the previous one, but this time having a semistable limit cycle of multiplicity 4 .

In this section $X=(P, Q)$ will denote the polynomial vector field of degree 11 given by

$$
\begin{aligned}
P= & 4 x-2 x^{2}-14 x^{3}+8 x^{4}+16 x^{5}-12 x^{6}-4 x^{7}+8 x^{8}-4 x^{9} \\
& -2 x^{10}+2 x^{11}+4 y-4 x y-30 x^{2} y+16 x^{3} y+24 x^{4} y-24 x^{5} y \\
& -13 x^{6} y+16 x^{7} y-3 x^{8} y-4 x^{9} y+3 x^{10} y-6 y^{2}-14 x y^{2} \\
& +48 x^{2} y^{2}+32 x^{3} y^{2}-72 x^{4} y^{2}-12 x^{5} y^{2}+60 x^{6} y^{2} \\
& -16 x^{7} y^{2}-18 x^{8} y^{2}+10 x^{9} y^{2}-30 y^{3}+16 x y^{3}+48 x^{2} y^{3} \\
& -48 x^{3} y^{3}-39 x^{4} y^{3}+48 x^{5} y^{3}-12 x^{6} y^{3}-16 x^{7} y^{3}+15 x^{8} y^{3} \\
& +40 y^{4}+16 x y^{4}-108 x^{2} y^{4}-12 x^{3} y^{4}+132 x^{4} y^{4}-24 x^{5} y^{4} \\
& -52 x^{6} y^{4}+20 x^{7} y^{4}+24 y^{5}-24 x y^{5}-39 x^{2} y^{5}+48 x^{3} y^{5} \\
& -18 x^{4} y^{5}-24 x^{5} y^{5}+30 x^{6} y^{5}-48 y^{6}-4 x y^{6}+116 x^{2} y^{6} \\
& -16 x^{3} y^{6}-68 x^{4} y^{6}+20 x^{5} y^{6}-13 y^{7}+16 x y^{7}-12 x^{2} y^{7} \\
& -16 x^{3} y^{7}+30 x^{4} y^{7}+36 y^{8}-4 x y^{8}-42 x^{2} y^{8}+10 x^{3} y^{8} \\
& -3 y^{9}-4 x y^{9}+15 x^{2} y^{9}-10 y^{10}+2 x y^{10}+3 y^{11} \\
Q & -4 x-2 x^{2}+30 x^{3}+8 x^{4}-24 x^{5}-12 x^{6}+13 x^{7}+8 x^{8}+3 x^{9} \\
& -2 x^{10}-3 x^{11}+4 y+4 x y-14 x^{2} y-32 x^{3} y+16 x^{4} y+36 x^{5} y \\
& -4 x^{6} y-28 x^{7} y-4 x^{8} y+8 x^{9} y+2 x^{10} y-6 y^{2}+30 x y^{2}+32 x^{2} y^{2} \\
& -48 x^{3} y^{2}-60 x^{4} y^{2}+39 x^{5} y^{2}+48 x^{6} y^{2}+12 x^{7} y^{2}-14 x^{8} y^{2} \\
& -15 x^{9} y^{2}-14 y^{3}-32 x y^{3}+32 x^{2} y^{3}+72 x^{3} y^{3}-12 x^{4} y^{3} \\
Q &
\end{aligned}
$$




$$
\begin{aligned}
& -84 x^{5} y^{3}-16 x^{6} y^{3}+32 x^{7} y^{3}+10 x^{8} y^{3}+24 y^{4}-24 x y^{4} \\
& -84 x^{2} y^{4}+39 x^{3} y^{4}+96 x^{4} y^{4}+18 x^{5} y^{4}-36 x^{6} y^{4}-30 x^{7} y^{4} \\
& +16 y^{5}+36 x y^{5}-12 x^{2} y^{5}-84 x^{3} y^{5}-24 x^{4} y^{5}+48 x^{5} y^{5} \\
& +20 x^{6} y^{5}-36 y^{6}+13 x y^{6}+80 x^{2} y^{6}+12 x^{3} y^{6}-44 x^{4} y^{6} \\
& -30 x^{5} y^{6}-4 y^{7}-28 x y^{7}-16 x^{2} y^{7}+32 x^{3} y^{7}+20 x^{4} y^{7}+24 y^{8} \\
& +3 x y^{8}-26 x^{2} y^{8}-15 x^{3} y^{8}-4 y^{9}+8 x y^{9}+10 x^{2} y^{9}-6 y^{10} \\
& -3 x y^{10}+2 y^{11}
\end{aligned}
$$

It is easy to check that

$$
\begin{aligned}
H= & \left(-1+x^{2}+y^{2}\right)^{-3}+2 \arctan \left(\frac{x}{-2+y}\right)+2 \arctan \left(\frac{x}{y}\right) \\
& +\log \left(x^{2}+y^{2}\right)+\log \left(4+x^{2}-4 y+y^{2}\right)+\log \left(\left|-1+x^{2}+y^{2}\right|\right)
\end{aligned}
$$

is a first integral of $X$; i.e.

$$
\frac{\partial H}{\partial x} P+\frac{\partial H}{\partial y} Q=0
$$

and consequently $H$ is constant on the orbits of the vector field $X$.

This system is already too complicated for P4 to study it but we know for sure (from the first integral) that it has a limit cycle of multiplicity 4 at the unity circle and thus we can do the same study as we have done up to now.

Since $f=x^{2}+y^{2}-1$ satisfies the equality

$$
\frac{\partial f}{\partial x} P+\frac{\partial f}{\partial y} Q=8\left(2-x+x^{2}-3 y+y^{2}\right)\left(x^{2}+y^{2}\right) f^{4},
$$

it follows that $f=0$ is an algebraic solution of $X$. Due to the fact that on $f=0$ there are no singular points, $f=0$ is a periodic orbit. Using the first integral we can see that it is isolated in the set of all periodic orbits, so it is a limit cycle, that we denote by $\Gamma$. Again, using the first integral, we can check that $\Gamma$ is a semistable limit cycle. Using results of [2] we can show that $\Gamma$ has multiplicity 4.

Repeating the arguments of Subsection 3.1, we obtain that the vector fields $X_{\mu}(x, y)=(P(x, y), Q(x, y)+\mu P(x, y))$ form a rotated family with respect 


\begin{tabular}{|c|c|c|c|}
\hline$n$ & $\mu_{n}$ & $\alpha_{n}$ & $C_{n}$ \\
\hline 1 & $-1,292490 \cdot 10^{-1}$ & & \\
2 & $-5,194300 \cdot 10^{-2}$ & $-1,315151$ & $-0,129249$ \\
3 & $-3,036667 \cdot 10^{-2}$ & $-1,323915$ & $-0,130036$ \\
4 & $-2,066923 \cdot 10^{-2}$ & $-1,337239$ & $-0,131954$ \\
5 & $-1,530841 \cdot 10^{-2}$ & $-1,345516$ & $-0,133477$ \\
& $\ldots \ldots \ldots \ldots$ & $\ldots \ldots$ & $\ldots \ldots$ \\
401 & $-4,005103 \cdot 10^{-5}$ & $-1,347385$ & $-0,128840$ \\
402 & $-3,991685 \cdot 10^{-5}$ & $-1,347405$ & $-0,128855$ \\
403 & $-3,978346 \cdot 10^{-5}$ & $-1,347267$ & $-0,128749$ \\
404 & $-3,965084 \cdot 10^{-5}$ & $-1,347350$ & $-0,128813$ \\
405 & $-3,951899 \cdot 10^{-5}$ & $-1,347251$ & $-0,128737$ \\
& $\ldots \ldots \ldots \ldots$ & $\ldots \ldots$ & $\ldots \ldots$ \\
\hline
\end{tabular}

Table 7 - Values of $\mu_{n}$ for the point $p_{0}=(0,1 / 2)$.

to the parameter $\mu \in \mathbb{R}$. Again, $X_{0}=X$, and $\mu=0$ is a bifurcation value for the parameter $\mu$ in such a way that for $\mu<0$ and small the vector field $X_{\mu}$ has no limit cycles. By the non-intersection property, there are no periodic orbits of $X_{\mu}$ in the region occupied by the period annulus around the infinity of system $X_{0}$.

We fix a point $p_{0}=\left(x_{0}, y_{0}\right)$ in $R=\left\{(x, y): 1 / 2 \leq x^{2}+y^{2}<1\right\}$, and uses as in the previous subsection the separatrix $\gamma_{\mu}$ in backward time for computing the sequence $\left(\mu_{n}\right)$.

Again for different points $p_{0}$, there is numerical evidence that the limit of $\alpha_{n}$ is $-4 / 3$ as $n \rightarrow \infty$ independently of the point $p_{0}$, because $\alpha_{404}=$ $-1,347251 \ldots$ If we start from $p=(0,3 / 4)$ (respectively $p=(0,7 / 8))$ we get that $\alpha_{404}=-1,344790 \ldots$ (respectively $\alpha_{404}=-1,327778 \ldots$ ).

\section{REFERENCES}

[1] M. Abramowitz and I.A. Stegun, Hypergeometric Functions. Ch. 15 in Handbook of Mathematical Functions with Formulas, Graphs, and Mathematical Tables, $9^{\text {th }}$ printing. New York: Dover (1972), 555-566. 
[2] C. Christopher, J. Llibre and J.V. Pereira, Multiplicity of Invariant Algebraic Curves in Polynomial Vector Fields. Pacific J. of Math., 229 (2007), 63-117.

[3] F. Dumortier, J. Llibre and J.C. Artés, Qualitative theory of planar differential systems. UniversiText, Springer-Verlag, New York (2006).

[4] M.J. Feigenbaum, Quantitative universality for a class of non-linear transformations. J. Statist. Phys., 19 (1978), 25-52.

[5] M.J. Feigenbaum, The universal metric properties of nonlinear transformations. J. Statist. Phys., 21 (1979), 669-706.

[6] Ye Yanqian, Theory of Limit Cycles. Translations of Math. Monographs, Amer. Math. Soc, Providence, 66 (1986). 